\title{
A unique haplotype of RCCX copy number variation: from the clinics of congenital adrenal hyperplasia to evolutionary genetics
}

\author{
Márton Doleschall ${ }^{\star 1,2,3}$, Andrea Luczay ${ }^{4}$, Klára Koncz ${ }^{5}$, Kinga Hadzsiev ${ }^{6}$, Éva Erhardt ${ }^{7}$, Ágnes Szilágyi ${ }^{3}$, \\ Zoltán Doleschall ${ }^{8}$, Krisztina Németh ${ }^{4}$, Dóra Török ${ }^{4}$, Zoltán Prohászka ${ }^{3}$, Balázs Gereben ${ }^{9}$, György Fekete ${ }^{4}$, \\ Edit Gláz ${ }^{5}$, Péter Igaz ${ }^{5}$, Márta Korbonits ${ }^{2}$, Miklós Tóth ${ }^{5}$, Károly Rácz ${ }^{1,5}$ and Attila Patócs ${ }^{1,10}$
}

There is a difficulty in the molecular diagnosis of congenital adrenal hyperplasia (CAH) due to the c.955C $>\mathrm{T}$ ( $\mathrm{p} .\left(\mathrm{Q} 319^{*}\right)$, formerly Q318X, rs7755898) variant of the CYP21A2 gene. Therefore, a systematic assessment of the genetic and evolutionary relationships between c.955C $>$ T, CYP21A2 haplotypes and the RCCX copy number variation (CNV) structures, which harbor CYP21A2, was performed. In total, 389 unrelated Hungarian individuals with European ancestry (164 healthy subjects, 125 patients with non-functioning adrenal incidentaloma and 100 patients with classical CAH) as well as 34 adrenocortical tumor specimens were studied using a set of experimental and bioinformatic methods. A unique, moderately frequent (2\%) haplotypic RCCX CNV structure with three repeated segments, abbreviated to LBSASB, harboring a CYP21A2 with a C.955C $>$ T variant in the 3 '-segment, and a second CYP21A2 with a specific c. ${ }^{*} 12 \mathrm{C}>\mathrm{T}$ (rs150697472) variant in the middle segment occurred in all c.955C $>$ T carriers with normal steroid levels. The second CYP21A2 was free of CAH-causing mutations and produced mRNA in the adrenal gland, confirming its functionality and ability to rescue the carriers from CAH. Neither LBSASB nor $c .{ }^{*} 12 \mathrm{C}>\mathrm{T}$ occurred in classical CAH patients. However, CAH-causing CYP21A2 haplotypes with c.955C $>$ T could be derived from the 3'segment of LBSASB after the loss of functional CYP21A2 from the middle segment. The $c .{ }^{*} 12 \mathrm{C}>\mathrm{T}$ indicated a functional CYP21A2 and could distinguish between non-pathogenic and pathogenic genomic contexts of the $c .955 \mathrm{C}>\mathrm{T}$ variant in the studied European population. Therefore, $c .{ }^{*} 12 \mathrm{C}>\mathrm{T}$ may be suitable as a marker to avoid this genetic confound and improve the diagnosis of CAH.

European Journal of Human Genetics (2017) 25, 702-710; doi:10.1038/ejhg.2017.38; published online 12 April 2017

\section{INTRODUCTION}

RCCX copy number variation $(\mathrm{CNV})$ is a complex (it contains another $\mathrm{CNV}$ ), multiallelic (there are more than two frequent $\mathrm{CNV}$ alleles in a population) and tandem (CNV segments are next to each other) CNV. ${ }^{1-3}$ Two full-length genes, complement component 4 (C4) and steroid 21-hydroxylase (CYP21), are located in RCCX CNV. C4 has two types with slightly different immune functions, ${ }^{4} C 4 A$ and $C 4 B$, and it contains an additional CNV, HERV-K(C4) CNV, in its intron 9 (Figure 1), derived from an ancient insertion of human endogenous retrovirus. RCCX CNV in one chromosome is referred as a haplotypic structure, and is traditionally described by the copy number of the segment, and, per segment, by the types of $C 4$ and the alleles of HERV-K(C4) CNV (see the explanation of more specialized genetic terms in Table 1). A haplotypic RCCX CNV structure usually contains one functional CYP21 gene (CYP21A2) in the $3^{\prime}$-segment, and zero, one or two disabled pseudogenes (CYP21A1P) in the segments towards the 5'-direction (Figure 1). CYP21A2 expresses the steroid 21-hydroxylase enzyme (cytochrome P450c21) uniquely in adrenal cortex, which is one of the enzymes responsible for the biosynthesis of the two principal steroid hormones, aldosterone and cortisol. ${ }^{5}$ Aldosterone regulates blood pressure by acting on kidney functions, and cortisol suppresses immune functions, responds to stress and takes part in the intermediary metabolism of different tissues. Two chromosomal copies of disabled CYP21A2 causes congenital adrenal hyperplasia (CAH; we use this broader, but more prevalent, term instead of 21-hydroxylase deficiency), an autosomal recessive disorder accompanied by a partial or complete lack of aldosterone and cortisol. ${ }^{6}$ Special genetic rearrangements generally featured by multiallelic CNVs, such as non-allelic gene conversion and unequal crossover, ${ }^{7}$ are responsible for the vast majority of CYP21A2 mutations leading to $\mathrm{CAH} ;{ }^{8}$ therefore, the complex genetic structure of RCCX $\mathrm{CNV}$ indirectly manifests in $\mathrm{CAH}$.

Difficulties in the molecular diagnosis of $\mathrm{CAH}$ due to the complicated genetic nature of RCCX CNV have been recognized for a long time, ${ }^{9}$ and the best-known case for this is an LSS haplotypic RCCX CNV structure with three segments harboring two copies of

${ }^{1}$ Molecular Medicine Research Group, Hungarian Academy of Sciences and Semmelweis University, Budapest, Hungary; ${ }^{2}$ William Harvey Research Institute, Barts and The London School of Medicine and Dentistry, Queen Mary University of London, London, UK; ${ }^{3} 3$ rd Department of Internal Medicine, Semmelweis University, Budapest, Hungary; ${ }^{4}$ 2nd Department of Pediatrics, Semmelweis University, Budapest, Hungary; ${ }^{5}$ 2nd Department of Internal Medicine, Semmelweis University, Budapest, Hungary; ${ }^{6}$ Department of Medical Genetics, University of Pecs, Pecs, Hungary; ${ }^{7}$ Department of Pediatrics, University of Pecs, Pecs, Hungary; ${ }^{8}$ Department of Pathogenetics, National Institute of Oncology, Budapest, Hungary; ${ }^{9}$ Department of Endocrine Neurobiology, Institute of Experimental Medicine, Hungarian Academy of Sciences, Budapest, Hungary; ${ }^{10}$ ‘Lendület’ Hereditary Endocrine Tumors Research Group, Hungarian Academy of Sciences and Semmelweis University, Budapest, Hungary

${ }^{*}$ Correspondence: Dr M Doleschall, Molecular Medicine Research Group, Hungarian Academy of Sciences and Semmelweis University, 46 Szentkiralyi Street, Budapest 1088 , Hungary. Tel: +36 305364111; Fax: +36 1 2660816; E-mail: doleschall.marton@med.semmelweis-univ.hu

Received 10 June 2016; revised 8 February 2017; accepted 14 February 2017; published online 12 April 2017 


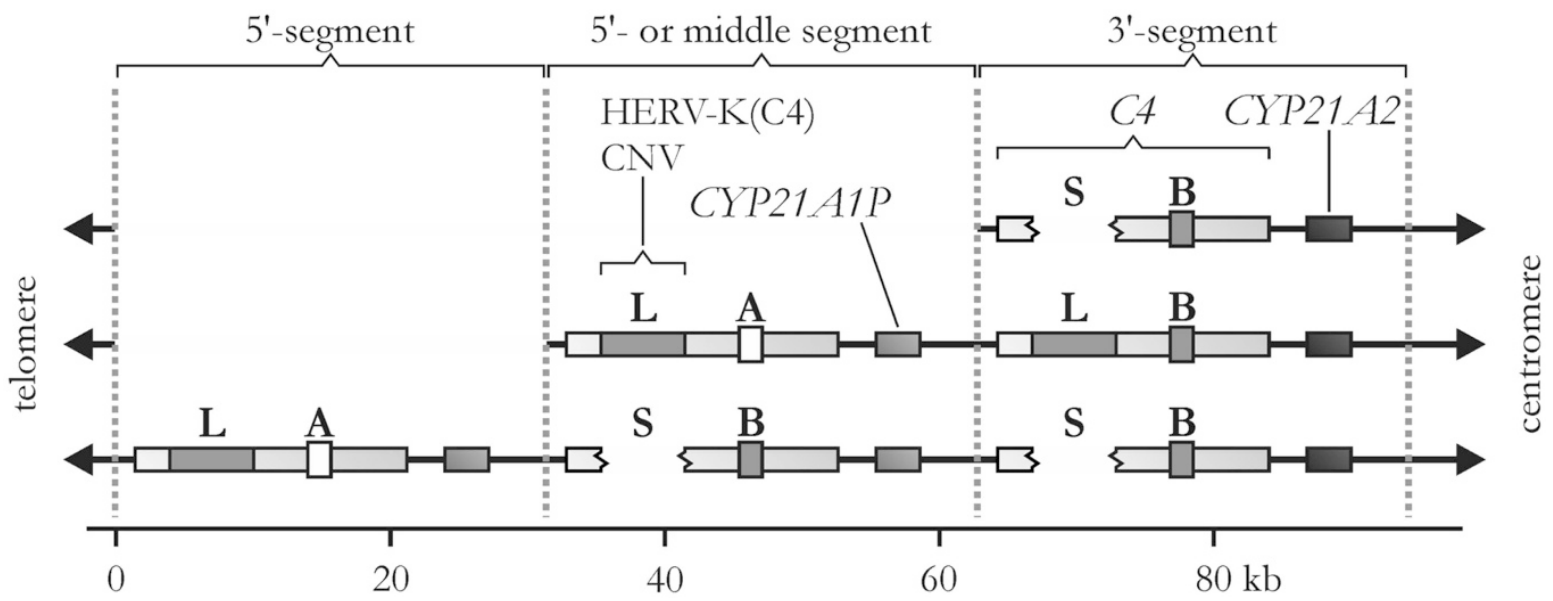

Figure 1 Scaled representation of the organization of human RCCX CNV depicted by RCCX structures with one, two and three segments. Each segment (repeat) is abbreviated with two letters, the first represents the alleles of the HERV-K(C4) CNV (L - long (insertion) allele or $\mathbf{S}$ - short (deletion) allele), and the second symbolizes the types of $C 4$ gene (A or B). The duplications of these two letters indicate RCCX structures with more than one segment. Dotted lines indicate the segment boundaries. The variable region of the RCCX with two segments contains two pairs of full-length genes, complement component 4 (C4A and C4B) and steroid 21-hydroxylase (CYP21A1P and CYP21A2). Other two gene pairs partially residing in RCCX CNV, TNXA-TNXB and STK19 and STK19P, are not illustrated.

Table 1 Glossary of genetic abbreviations and key terms used in the text of the current study

\begin{tabular}{|c|c|c|}
\hline \multirow[t]{5}{*}{ CNV } & Copy number variation & $\begin{array}{l}\text { A form of structural variations where a longer DNA segment occurs with different repeat numbers in the } \\
\text { different copies of the same homolog chromosome; thus, the copy number of the DNA segment will vary } \\
\text { among chromosomes and individuals. }\end{array}$ \\
\hline & CNV allele & A particular number of repeated segments in one chromosome. \\
\hline & CYP21A2 haplotype & $\begin{array}{l}\text { A particular combination of the alleles of all variations (SNPs and mutations) in CYP21A2 of one } \\
\text { chromosome. It is identical to a Mendelian allele of the full-length CYP21A2. }\end{array}$ \\
\hline & Haplotype & $\begin{array}{l}\text { A particular combination of the alleles of adjacent genetic variants in one chromosome, which are not } \\
\text { independent of each other, and are frequently inherited together. }\end{array}$ \\
\hline & $\begin{array}{l}\text { Haplotypic RCCX CNV structure (its short } \\
\text { form is RCCX structure) }\end{array}$ & $\begin{array}{l}\text { A complex variant in one chromosome which is traditionally described by the copy number of the repeated segment } \\
\text { of RCCX CNV (CNV allele), and, per segment, by the alleles of HERV-K (C4) CNV and the type of C4 gene. }\end{array}$ \\
\hline HERV-K(C4) & Human endogenous retrovirus- $K$ & An ancient genomic remnant of a human endogenous retrovirus- $K$ in the intron 9 of human $C 4$ genes. \\
\hline HERV-K(C4) CNV & & $\begin{array}{l}\text { A CNV with a deletion or insertion CNV allele (null or one segment) in a } C 4 \text { gene of one chromosome derived } \\
\text { from a human endogenous retrovirus-K. }\end{array}$ \\
\hline LASA & LASA RCCX structure & $\begin{array}{l}\text { A RCCX structure with two segments containing a } C 4 A \text { with HERV-K(C4) insertion in the } 5^{\prime} \text {-segment and a } \\
C 4 A \text { without HERV-K(C4) in the } 3^{\prime} \text {-segment. }\end{array}$ \\
\hline LBSASB & LBSASB RCCX structure & $\begin{array}{l}\text { A RCCX structure with three segments containing a } C 4 B \text { with HERV-K(C4) insertion in the } 5^{\prime} \text {-segment, a } C 4 A \\
\text { without HERV-K(C4) in the middle segment and a } C 4 A \text { without HERV-K(C4) in the } 3^{\prime} \text {-segment. }\end{array}$ \\
\hline LD & Linkage disequilibrium & $\begin{array}{l}\text { A statistical term where two or more alleles of adjacent variants tend to be associated with each other. It is a } \\
\text { statistical representation of the haplotypes within a particular population. }\end{array}$ \\
\hline LSS & LSS RCCX structure & $\begin{array}{l}\text { A RCCX structure with three segments, where the types of } C 4 \text { are not determined, contains a HERV-K(C4) } \\
\text { insertion only in the } 5 \text { '-segment. }\end{array}$ \\
\hline RCCX CNV & & A CNV of chromosome 6 , which was named after the traditional name of its gene contents. \\
\hline SB & SB RCCX structure & A RCCX structure with one segment containing a $C 4 B$ without HERV-K(C4) insertion in its single segment. \\
\hline
\end{tabular}

Abbreviations: CNV, copy number variation; SNP, single-nucleotide polymorphism.

CYP21A2 and the c.955C > T (p. (Q319*), rs7755898) variant. (For numbering the genetic variants, we use the most recent human reference gene and the corresponding mRNA sequence (RefSeqGene NG_007941.3 and NM_000500.7), which contains the insertion (CTG) allele of the c.28_30delCTG (rs61338903) variant. Therefore, our numbering differs from the studies using a CYP21A2 sequence (eg, former RefSeqGene NG_007941.2) with the deletion allele of c.28_30delCTG. The p. $\left(\mathrm{Q} 319^{*}\right)$ variant name is used with parentheses at the protein level according to the nomenclature recommendation of
Human Genome Variation Society (http://varnomen.hgvs.org/), which considers a protein variant without experimental evidence at RNA or protein level as a predicted one; however, the segregation patterns of classical (severe form) CAH families ${ }^{8,10-12}$ (especially patients from consanguineous families with family history having homozygous c.955T genotypes without any other $\mathrm{CAH}$ mutations in exons and exon/intron boundaries and without reported duplicated CYP21A2 ${ }^{13}$ ) have provided clinical evidences for the pathogenicity since the initial report of $\mathrm{c} .955 \mathrm{C}>\mathrm{T}^{14}$ ) This unique RCCX structure was found for 


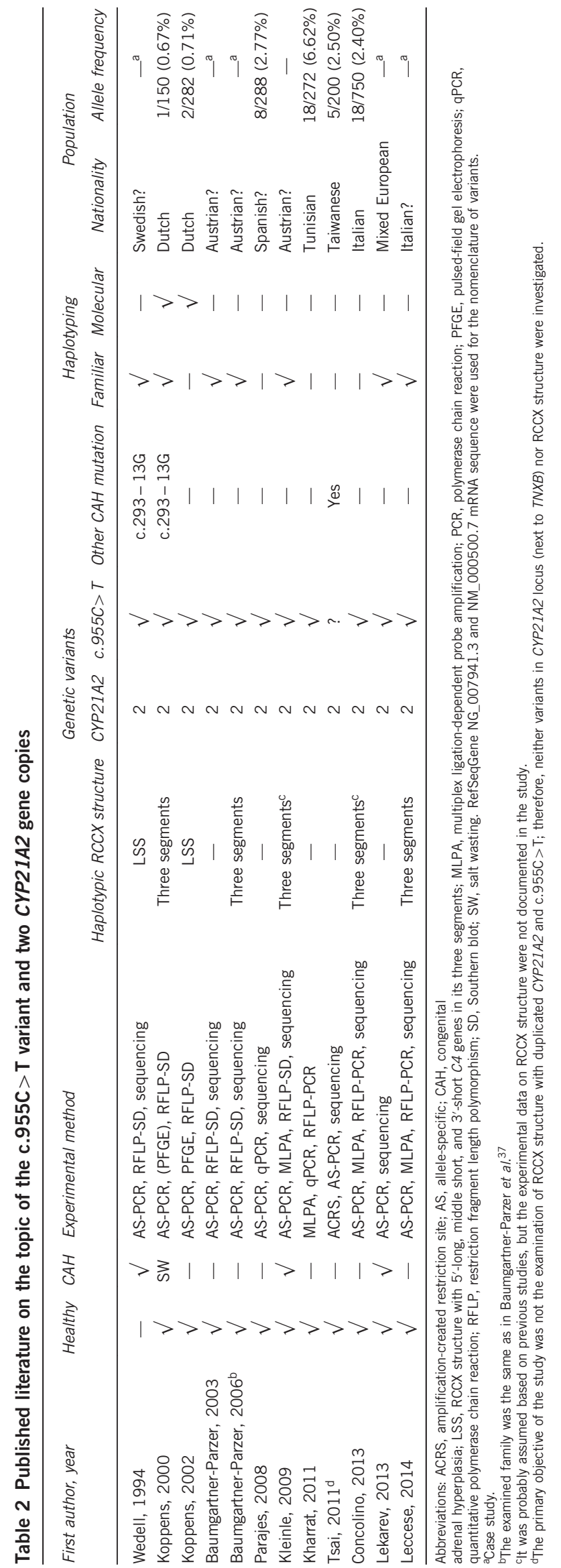

the first time in three patients with $\mathrm{CAH}$, and the c.293-13C $>\mathrm{R}$ (rs6467) CAH mutation accompanied the c.955C $>\mathrm{T}$ variant. ${ }^{10}$ The unique RCCX structure with c.955C > T and c.293-13C > R has been confirmed in a Dutch CAH population, ${ }^{15}$ but the same RCCX structure without the c.293-13C > R has been observed in the healthy subjects of a Dutch population as well. ${ }^{16}$ After the initial findings, several studies on the topic have been published over time. However, healthy and $\mathrm{CAH}$ populations have rarely been examined simultaneously, and the determination of underlying RCCX structures has often been incomplete or has not been documented (Table 2). Nevertheless, new information on the unique haplotypic RCCX structure has been accumulated. In a healthy Spanish population, two CYP21A2 gene copies in one chromosome are associated with three genetic variants in complete linkage disequilibrium (LD): c. $955 \mathrm{C}>\mathrm{T}, \quad$ c. $293-79 \mathrm{G}>\mathrm{A} \quad(\mathrm{rs} 114414746)$ and c. ${ }^{\star} 12 \mathrm{C}>\mathrm{T}$ (rs150697472) variants. They occur with a moderate allele frequency (2.77\%), and c.955C $>\mathrm{T}$ is located in the $3^{\prime}$-segment of CYP21A2, whereas a wild-type CYP21A2 is in the middle segment. ${ }^{17} \mathrm{An}$

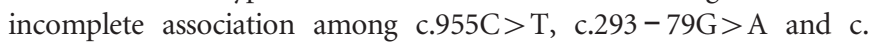
${ }^{\star} 12 \mathrm{C}>\mathrm{T}$ has also been found in a later study, ${ }^{18}$ but, unfortunately, the diagnoses of $\mathrm{CAH}$ in the studied individuals have not been clearly documented, and therefore it cannot be known if the presence or absence of the association has been assigned to patients with CAH or healthy subjects.

In the current study, a healthy and a CAH population with the same European ancestry were used to assess the occurrences and association of the haplotypic RCCX structure with three segments, two CYP21A2 in the same RCCX structure, c.955C $>\mathrm{T}, \mathrm{c.293-79G>A}$ and c. ${ }^{\star} 12 \mathrm{C}>\mathrm{T}$, and to offer a solution for the diagnostic problem. A recent set of experimental and bioinformatic methods ${ }^{1,19}$ were applied to decipher the exact haplotypes of RCCX structure and CYP21A2, and to translate the evolutionary origin of the studied genetic variants into clinically useful information. Furthermore, patients with nonfunctioning adrenal incidentaloma (NFAI), which is a hormonally inactive adrenal mass unintentionally discovered by medical imaging, ${ }^{20}$ and the adrenocortical tumor specimens of NFAI patients, were used to compare blood hormone levels and to examine the mRNA levels. The rationale for studying NFAI patients was: (i) the baseline hormone profiles of NFAI subjects do not differ from those of healthy subjects, (ii) the NFAI subjects had a broader hormone profile and (iii) their tumor specimens are available.

\section{MATERIALS AND METHODS}

\section{Subjects and clinical data}

For the subjects with the c.955C > T variant, 96 healthy subjects (50\% women) from a family study, ${ }^{21} 68$ healthy subjects $(65.2 \%$ women $)$ and 125 patients (76.0\% women) with NFAI from a recent study, ${ }^{22}$ and 100 patients $(58.0 \%$ women) with classical CAH due to 21-hydroxylase deficiency from the Second Department of Internal Medicine, Semmelweis University (Budapest, Hungary) and the Second Department of Pediatrics, Semmelweis University (Budapest, Hungary) were screened. In addition, one patient with supposed classical CAH was referred to us by the Department of Pediatrics, University of Pecs (Pecs, Hungary) for reexamination of the original genetic diagnosis. All subjects were genetically unrelated and Hungarian with European ancestry. The exclusion criteria for NFAI patients were described recently. ${ }^{22}$ The diagnosis was confirmed in all CAH patients by hormonal and genetic testing (except for the one who was reexamined) as described. ${ }^{23,24}$ Furthermore, 34 adrenocortical tumor specimens of NFAI patients were obtained as described, ${ }^{25}$ and screened for the c.955C $>\mathrm{T}$ variant. All subjects gave written informed consent. The study protocol was approved by the National Scientific and Ethical Committee, Medical Research Council of Hungary (TUKEB, ETT), and was executed according to the Declaration of Helsinki principles. 

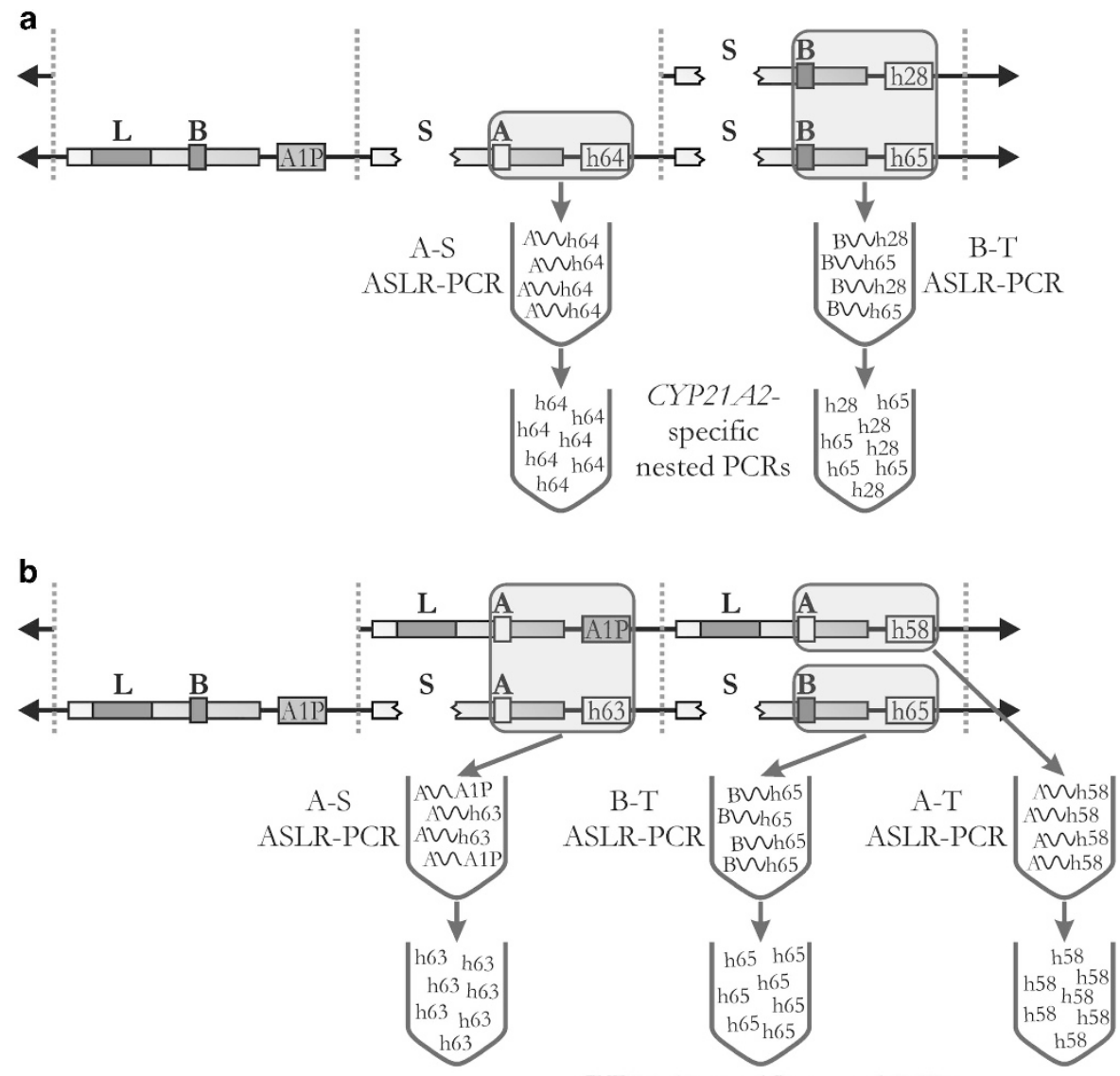

CYP21A2-specific nested PCRs

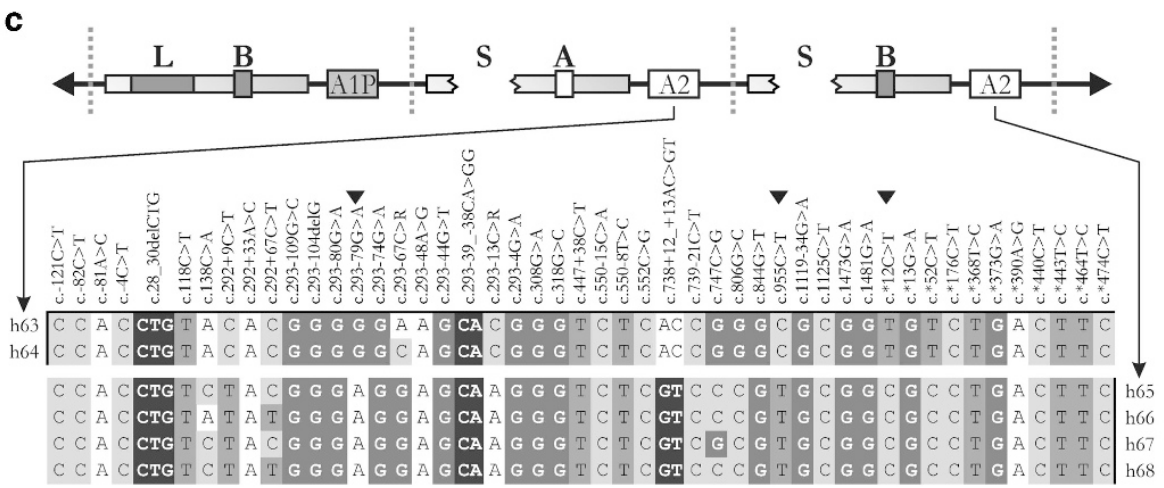

Figure 2 The experimental determination (molecular haplotyping) of haplotypic RCCX CNV structures, CYP21A2 haplotypes and the resultant CYP21A2 haplotypes of LBSASB RCCX structure. A-S ASLR-PCR indicates allele-specific long-range PCR only from the $C 4 A$ gene in the $5^{\prime}$-segment or the middle segment, A-T ASLR-PCR indicates ASLR-PCR only from the C4A gene in the $3^{\prime}$-segment, and B-T ASLR-PCR indicates ASLR-PCR only from the C4B gene in the 3'-segment. Each haplotypic RCCX segment is abbreviated with two letters, the first represents the alleles of HERV-K(C4) CNV (L - long (insertion) allele or $\mathbf{S}$ - short (deletion) allele), and the second symbolizes the types of $C 4$ gene (A or $\mathbf{B})$. The multiplication of the two letters in a structure indicates the segment number. Other two gene pairs partially residing in RCCX CNV, TNXA-TNXB and STK19 and STK19P, are not illustrated. (a) The haplotypic RCCX structures and the CYP21A2 haplotype in the middle segment can be determined from the depicted diploid combination of the haplotypic RCCX structures, but the CYP21A2 haplotype in the 3'-segment cannot. (b) All CYP21A2 haplotypes can be determined from the depicted diploid combination of the haplotypic RCCX structures, because ASLR-PCR can separate the two 3'-segments from the different haploid copies of chromosome 6. (c) CYP21A2 haplotypes of the LBSASB RCCX structure. Only variants with at least moderate frequencies according to a recent study ${ }^{19}$ are presented. Specific genetic variants of these haplotypes are indicated by black triangles.

\section{Genetic analyses of RCCX CNV and CYP21 genes}

The c.955T allele, full haplotypic RCCX CNV structures, and full-length CYP21A1P and CYP21A2 haplotypes were determined as described previously. ${ }^{1,19,22-24}$ Briefly, a combined molecular and inferred haplotyping approach was used with two stages. C4 type-specific quantitative PCRs
(qPCRs), CYP21 gene-specific qPCR, HERV-K(C4) CNV allele-specific qPCR, a set of CYP21 gene-specific nested polymerase chain reactions (PCRs) resting on a set of allele-specific long-range (ASLR) PCRs, and the Sanger sequencing of the nested PCR products were carried out in the first, experimental stage. One smaller modification was set up using a new CYP21A1P-specific nested 
PCR primer for one individual, CYP21A1P_2R (Supplementary Table 1), because a mutation of CYP21A1P decreased the binding efficacy of the original primer, CYP21A1P_R. ${ }^{1}$ The experimentally determined new CYP21A1P and CYP21A2 haplotypes are available in GenBank (www.ncbi.nlm.nih.gov/genbank/) under KU302771-KU302776 IDs, and variants (IDs in Supplementary Table 2) in dbSNP (www.ncbi.nlm.nih.gov/projects/SNP/). The abovementioned haplotypic genetic features of RCCX CNV and CYP21 genes, which could not be determined in some subjects, were inferred by bioinformatic haplotype reconstruction in the second stage. To map the origin of haplotypic RCCX structures and CYP21A2 haplotypes, a genealogical haplotype network was constructed using the new and published CYP21A2 haplotypes (Supplementary Table 2 and 3 ) as described. ${ }^{1}$

\section{Functional studies on CYP21A2}

Total RNA was isolated from adrenocortical tumor specimens by RNeasy Lipid Tissue Kit (Qiagen, Hilden, Germany), cDNA was synthesized by ProtoScript II Kit (New England Biolabs, Ipswich, MA, USA) and CYP21A2 mRNA level was determined by qPCR using a combined approach as described. ${ }^{19,26}$ Briefly, the forward primer was designed on the boundary of exons 1 and 2 of the CYP21 genes, the reverse primer was located on c.332_339delGAGACTAC variant distinguishing CYP21A1P and CYP21A2 and the Taqman minor groove binder probe (Applied Biosystems, Foster City, CA, USA) on the boundary of exons 2 and 3 was labeled with the fluorescent dye 6-FAM (Supplementary Table 1). Reactions were carried out in a 7500 Fast Real-Time PCR equipment by TaqMan Fast Advanced PCR master mix (Applied Biosystems). The human $O A Z 1$ gene was used as an internal control. ${ }^{27}$ Full-length CYP21 cDNA was amplified and cloned with primers designed for mRNA according to a previous study. ${ }^{28}$ Briefly, CYP21A2 cDNA was amplified by Phusion DNA polymerase (New England Biolabs), the PCR product was directionally cloned into a pTZ57R vector and subjected to Sanger sequencing. The methods of hormone measurements for NFAI patients and the software for statistics were recently described. $^{22}$

\section{RESULTS}

A unique haplotypic RCCX CNV structure with three segments, LBSASB, harboring two CYP21A2 gene copies and the c.955C $>$ T variant

The c.955C $>$ T variant was detected in 7 healthy subjects out of 164 (allele frequency is $2.1 \%$ ), in 5 NFAI patients out of $125(2.0 \%)$, and in 6 classical CAH patients out of 100 patients (3.0\%). To find out which haplotypic RCCX CNV structure and CYP21A2 haplotype harbor the c.955T allele, a set of molecular haplotyping methods ${ }^{1}$ were used for the experimental dissection in the first stage. Then, the haplotypic information based on molecular haplotyping from the first stage and the genotypic information of all studied healthy subjects and patients with NFAI were used for bioinformatic haplotype reconstruction in the second stage (see detailed description of how the experimental and bioinformatic methods work elsewhere ${ }^{1}$ ). The first, experimental stage revealed the following genetic features: (i) All the healthy subjects and all the NFAI patients who had the c.955T allele (12 individuals) carried a haplotypic RCCX CNV structure on one chromosome harboring two CYP21A2 gene copies (they had three CYP21A2 gene copies as a whole). (ii) The extra CYP21A2 gene copy in the $5^{\prime}$-segment or the middle segment of RCCX CNV was always related to a C4A gene in the $5^{\prime}$-direction, and the CYP21A2 haplotype of these segments could be determined in all 13 individuals (Figure 2a). Accordingly, two almost identical and unique (not observed in former studies ${ }^{1,19}$ ) CYP21A2 haplotypes were located in the $5^{\prime}$-segment or the middle segment, the h63 (in 1 individual) and h64 (in 11 individuals). (iii) From these 11 individuals with the h64 haplotype, the middle segment of an LBSASB RCCX structure harbored h64 in two individuals. (iv) Two individuals with c. $955 \mathrm{C}>\mathrm{T}$ carried a suitable RCCX structure combination for the CYP21A2 haplotype determination in the 3 -segment (Figure $2 \mathrm{~b}$ ), and they had a unique, undescribed h65 CYP21A2 haplotype there. In addition, both CYP21A2 haplotypes in one haplotypic RCCX structure were also determined in a 1-year-old $\mathrm{CAH}$ patient with c.955C $>\mathrm{T}$, whose DNA sample was received to reexamine the original genetic diagnosis based on parental genotypes, because the required dose of steroid substitution was unchanged in spite of weight gain. In this putative $\mathrm{CAH}$ patient, the 3 '-segment also harbored a h65 haplotype, and a h64 haplotype was carried as a second CYP21A2 copy.

The second, bioinformatic stage resolved a unique LBSASB RCCX structure harboring very similar h63 or h64 CYP21A2 haplotypes in the middle segment and very similar h65, h66, h67 or h68 haplotypes in the 3 '-segment above a 0.99 confidence probability threshold in all 12 individuals and the reexamined $\mathrm{CAH}$ patient (Figure 2c). A specific allele, c. ${ }^{\star} 12 \mathrm{~T}$, occurred only in h63 and h64 haplotypes of the middle segment and not in any other haplotypes of healthy subjects and patients with NFAI, and a specific allele, c.293-79A, occurred only in h65-h68 haplotypes of the $3^{\prime}$-segment (Supplementary Table 2).

Table 3 Haplotypes and genotypes of classical CAH patients with the c.955C $>$ T mutation

\begin{tabular}{|c|c|c|c|c|c|c|c|}
\hline \multirow{2}{*}{ Patient ID } & \multirow{2}{*}{ RCCX structure } & \multicolumn{6}{|c|}{ CYP21 genes } \\
\hline & & 5'-Segment & 3'-Segment & Classical CAH mutation in $3^{\prime}$-segment & Haplotype & c. $293-79 A$ & c. ${ }^{*} 12 T$ \\
\hline \multirow[t]{2}{*}{ Patient 1} & LALB & A1P & A2 & c.332_339delGAGACTAC & Det & $\mathrm{N}$ & $\mathrm{N}$ \\
\hline & LALB & A1P & 5'-A2 and 3'-A1P (intron 6) & c. $955 \mathrm{C}>\mathrm{T}$ & h69 & $\mathrm{N}$ & $\mathrm{N}$ \\
\hline \multirow[t]{2}{*}{ Patient 2} & LASB & A1P & A2 & c. $293-13 C>A, G$ & - & $Y$ (het) & $\mathrm{N}$ \\
\hline & LASB & & & c. $955 \mathrm{C}>\mathrm{T}$ & & & \\
\hline \multirow[t]{2}{*}{ Patient 3} & LASB & A1P & A2 & c. $293-13 C>A, G$ & - & $\mathrm{N}$ & $\mathrm{N}$ \\
\hline & LA?B & & & c. $955 \mathrm{C}>\mathrm{T}$ & & & \\
\hline \multirow[t]{2}{*}{ Patient 4} & LA & - & A1P & - & Det & $\mathrm{N}$ & $\mathrm{N}$ \\
\hline & LASB & A1P & $\mathrm{A} 2$ & c. $955 \mathrm{C}>\mathrm{T}$ & h70 & $\mathrm{N}$ & $\mathrm{N}$ \\
\hline \multirow[t]{2}{*}{ Patient 5} & ?BSA & $A 1 P$ & A2 & c. $955 \mathrm{C}>\mathrm{T}$ & - & $Y$ (het) & $\mathrm{N}$ \\
\hline & ??LA & & $5^{\prime}-\mathrm{A} 2$ and $3^{\prime}-\mathrm{A} 1 \mathrm{P}$ (intron 4?) & c. $955 \mathrm{C}>\mathrm{T}$ & & & \\
\hline \multirow[t]{2}{*}{ Patient 6} & LASA & A1P & A2 & c. $955 \mathrm{C}>\mathrm{T}$ & h65 & Y & $\mathrm{N}$ \\
\hline & ??LB & $?$ & $\mathrm{~A} 2$ & c.332_339delGAGACTAC & Det & $\mathrm{N}$ & $\mathrm{N}$ \\
\hline
\end{tabular}

Abbreviations: Det, determined haplotypes; het, in heterozygous form; $\mathrm{N}$, it does not contain the particular allele; $\mathrm{Y}$, it contains the particular allele.

Each haplotypic RCCX segment is abbreviated with two letters, the first represents the alleles of HERV-K(C4) CNV ( $\mathrm{L}-$ long allele or $\mathrm{S}-$ short allele), and the second symbolizes the types of $C 4$ gene $(A$ or $B)$. The multiplication of the two letters in a structure indicates the segment number. Question mark (?) indicates uncertainty in the particular genetic feature. RefSeqGene

NG_007941.3 and NM_000500.7 mRNA sequence were used for the nomenclature of variants. 
Consequently, these two variants and c.955C $>\mathrm{T}$ have the same allele frequency as the LBSASB RCCX structure harboring them, they were in complete $\operatorname{LD}\left(r^{2}=1\right)$, and, furthermore, there was no significant difference between the allele frequencies in healthy subjects and patients with NFAI (Fisher's exact test, $P=1.0000$ ).

CYP21A2 haplotypes of classical CAH patients with c.955C $>\mathrm{T}$ In total, seven CYP21A2 haplotypes with the c.955T allele were found in six patients out of the studied 100 patients with classical CAH (Table 3 ). The c. $^{\star} 12 \mathrm{~T}$ allele did not occur in these six CAH patients, but the c.293-79A allele was observed in three patients. From the seven haplotypes with the c.955T allele, three CYP21A2 haplotypes were experimentally determined; a h65 haplotype harbored by an LASA RCCX CNV structure, a haplotype (h69), which had an extended CYP21A1P sequence approximately from intron 6 to the $3^{\prime}$ end of the gene including c.955T, and a haplotype (h70), which did not harbor any alleles characterizing CYP21A1P apart from c.955T. Bioinformatic haplotype reconstruction can accurately resolve haplotypes when they recurrently occur in a studied population, but CYP21A2 haplotypes with CAH variants not determined by experiments did not satisfy this requirement. However, it was highly probable that a CYP21A2 haplotype (in patient 2) was identical with the h67 haplotype of LBSASB RCCX structure, and a haplotype (in patient 5) also had an extended CYP21A1P sequence at the $3^{\prime}$ end of the gene. Furthermore, the CYP21A2 haplotypes of CAH patients were not assigned to LBSASB or a chromosome harboring a second copy of CYP21A2, although the presence of the c.293-79A allele in classical $\mathrm{CAH}$ patients implied that the CYP21A2 haplotypes harboring c.293-79A had derived from the CYP21A2 in the $3^{\prime}$-segment of LBSASB.

Origin of CYP21A2 haplotypes with c.955C $>\mathrm{T}$ in healthy and $\mathrm{CAH}$ individuals

To assess the evolutionary origin of CYP21A2 haplotypes, a genealogical (evolutionary) haplotype network was applied as described recently. ${ }^{1}$ Briefly, haplotypes that are more similar to each other are located close to each other in the haplotype network, and are considered to be closer relatives. ${ }^{29}$

The CYP21A2 haplotypes (h63 and h64) in the middle segment of the LBSASB RCCX structure clustered together and were far from the group of the haplotypes (h65-h68) in the $3^{\prime}$-segment of LBSASB on the haplotype network (Figure 3a). This supports the idea that the haplotypes in each group were closely linked, but a distant relationship was observed between the two groups. The distant relationship implies that an unequal crossover event between two distantly related RCCX structures generated the LBSASB structure. However, the SB and LASB RCCX structure groups next to the RCCX structure assigned to h63h64 and h65-h68 groups do not suggest a one-step mechanism for this structure generation. Nevertheless, a potential multistep generation with unequal crossover or an alternative explanation of multiple non-allelic gene conversion events equally indicates a relatively ancient origin of the LBSASB structure. Not only the multistep mechanism but the presence of LBSASB-specific variants also confirms this.

One experimentally determined CYP21A2 haplotype with the c.955T allele from classical CAH patients was in the haplotype group of the 3 '-segment of the LBSASB, indicating a common descent. The LASA RCCX structure harboring the LBSASB-identical h65 haplotype was generated by simple (equal) or unequal crossover $^{1,30}$ between LBSASB and existing RCCX structures in the studied population. ${ }^{1,21}$ In the case of (equal) crossover, the breakpoint would have been after the $C 4 A$ in the $3^{\prime}$-segments of the LASA structure and between the
C4B and CYP21A2 in the $3^{\prime}$-segment of the LBSASB harboring the h65 haplotype (Figure $3 \mathrm{~b}$ ), while it would have been after the $C 4 A$ in the middle segments of the LASALB structure and in the same place of the LBSASB structure in the case of unequal crossover. In both cases, the functional CYP21A2 in the middle segment of LBSASB vanished from the emerged LASA RCCX structure.

Two other experimentally determined CAH-causing CYP21A2 haplotypes with the c.955T allele (h69 and h70) were located separately from either haplotypes in LBSASB and from each other, supporting their independent origins from the LBSASB RCCX structure (probably by non-allelic gene conversions from the CYP21A1P of the same RCCX structure where c.955T occurs). The connections of these two CYP21A2 haplotypes to the stem of the haplotype network were confirmed by the harboring RCCX structures of the connected haplotypes (h69 harbored by LALB RCCX structure and h70 harbored by LASB RCCX structure).

\section{Gene expression and function of CYP21A2 in the carriers of} LBSASB RCCX structure with the c.955C $>\mathrm{T}$ variant

LBSASB RCCX structure with the c.955T allele was observed in 1 out of 34 adrenocortical tumor specimens of patients with NFAI. The CYP21A2 mRNA level of this c.955C $>\mathrm{T}$ carrier patient did not significantly differ ( $t$-test, $P=0.3312$ ) from those of non-carrier patients (Figure 4a). Furthermore, this c.955C $>\mathrm{T}$ carrier patient had an h64 CYP21A2 haplotype in the middle segment of LBSASB, an h65 haplotype in the $3^{\prime}$-segment, and an h28 haplotype in the SB RCCX structure of the other chromosome (the exact RCCX structures of this patient is actually shown in Figure 2a). The transcripts of these three CYP21A2 haplotypes harbored distinctive alleles on $\mathrm{c}^{*} 12 \mathrm{C}>\mathrm{T}$ and c. ${ }^{\star} 52 \mathrm{C}>\mathrm{T}$ variants; CC alleles for h65, CT alleles for h28 and TT alleles for h64 (Figure $4 \mathrm{~b}$ ). To ascertain the frequencies of the different transcripts derived from these three different CYP21A2 haplotypes, cDNA was generated from total mRNA, and the CYP21A2 transcripts were cloned and sequenced in five independent experiments. The h64 transcript was present, indicating that CYP21A2 in the middle segment was transcriptionally active. The h65 transcript was not detected, whereas the frequency of the h28 transcript from the other chromosome was significantly lower than that of the h64 transcript (Figure 4c). The hormone profiles of the carriers of LBSASB with c. $955 \mathrm{C}>\mathrm{T}(N=5)$ and non-carriers $(N=102)$ of NFAI patients from our recent study ${ }^{22}$ were compared, but no significant difference was detected in morning cortisol, midnight cortisol, adrenocorticotropic hormone (ACTH)-induced cortisol, morning aldosterone, ACTHinduced aldosterone, morning 17-OH-progesterone, morning corticosterone, ACTH-induced corticosterone, metyrapone-blocked 11deoxycortisol, morning dehydroepiandrosterone sulfate, ACTH, or metyrapone-blocked ACTH levels (Supplementary Table 4).

\section{DISCUSSION}

A moderately frequent, unique haplotypic RCCX CNV structure with three segments harboring two CYP21A2 gene copies, abbreviated to LBSASB, was determined in 13 individuals (seven healthy subjects, five patients with NFAI and one reexamined CAH patient) with normal steroid levels by a set of experimental and bioinformatic methods published recently. ${ }^{1,19}$ This set of methods is capable of not only a more exact determination of RCCX structure than some current methods but also the segment-specific sequencing of CYP21A2: thus the haplotypes of the two harbored CYP21A2 in the middle and the $3^{\prime}$ segments of the RCCX CNV were revealed. The CYP21A2 haplotypes in the middle segment harbored the c. $^{\star} 12 \mathrm{~T}$ allele, whereas the CYP21A2 haplotypes in the $3^{\prime}$-segment harbored the c.293-79A and 


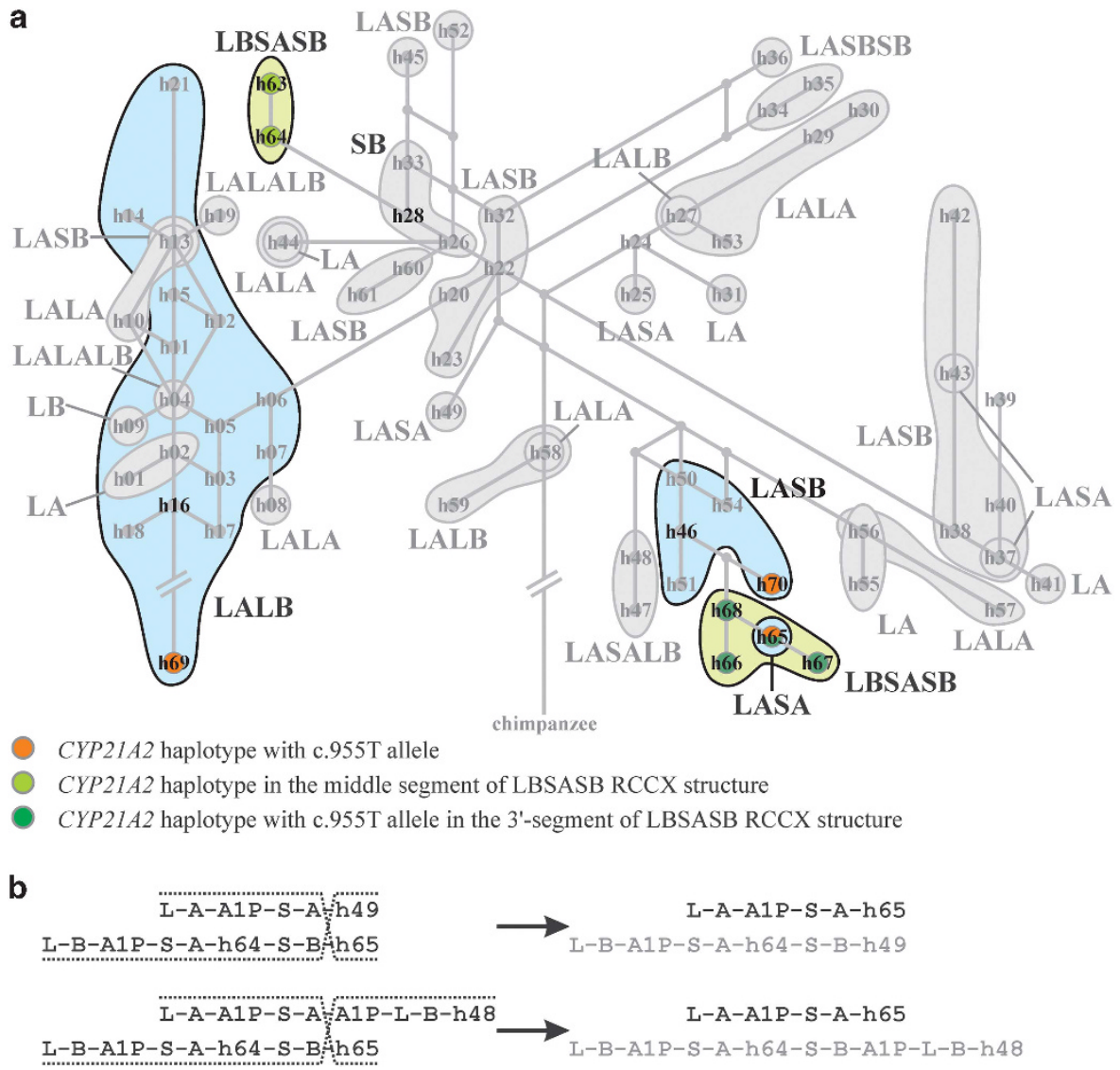

Figure 3 Evolutionary processes of RCXX CNV structure harboring the C.955C>T variant in CYP21A2. Each haplotypic RCCX segment is abbreviated with two letters, the first represents the alleles of HERV-K(C4) CNV ( $\mathbf{L}$ - long (insertion) allele or $\mathbf{S}$ - short (deletion) allele), and the second symbolizes the types of $C 4$ gene (A or B). The multiplication of the two letters in a structure indicates the segment number. (a) Genealogical haplotype networks of CYP21A2 haplotypes with projected haplotypic RCCX CNV structures. Numbers in gray circles indicate the known CYP21A2 haplotype, and numbers in orange, green, and dark green circles represent the CYP21A2 haplotype published here for the first time. Orange circles indicate CYP21A2 haplotypes with the C.955C $>$ T variant in classical CAH patients, green circles represent CYP21A2 haplotypes in the middle segment of the LBSASB RCCX structure, and dark green circles indicate CYP21A2 haplotypes with the c.955C > T in the $3^{\prime}$-segment of LBSASB. The length of gray lines between the CYP21A2 haplotypes is proportional with the nucleotide (allele) identity: greater length implies more different alleles between haplotypes and lower identity. (b) Both simple (equal) crossover between LASA and LBSASB RCCX structures and unequal crossover between LASALB and LBSASB structures could generate the LASA structure harboring a h65 CYP21A2 haplotype in the 3'-segment.

c.955T alleles. Both c. $293-79 \mathrm{G}>\mathrm{A}$ and c. $^{\star} 12 \mathrm{C}>\mathrm{T}$ variants are in complete $\mathrm{LD}$ with the $\mathrm{c} .955 \mathrm{C}>\mathrm{T}$ variant in the healthy subjects of two European (Spanish and Italian) populations, ${ }^{17,31}$ suggesting a straightforward usage of these variants to distinguish between pathogenic and non-pathogenic genomic contexts of the c.955C $>\mathrm{T}$ variant in the genetic diagnosis of $\mathrm{CAH}$, but unfortunately there is no available information from c. $293-79 \mathrm{G}>\mathrm{A}$ and c. ${ }^{*} 12 \mathrm{C}>\mathrm{T}$ in the $\mathrm{CAH}$ patients of these populations. Our findings on the healthy subjects and the patients with NFAI and CAH of the Hungarian population indicated that only the c. ${ }^{*} 12 \mathrm{~T}$ allele is unique in the individuals with normal steroid hormone levels. Therefore, the genetic examination of c. ${ }^{\star} 12 \mathrm{C}>\mathrm{T}$ allows us to avoid the genetic misinterpretation of p. $\left(\mathrm{Q} 319^{*}\right)$.

The ability of the c. ${ }^{*} 12 \mathrm{C}>\mathrm{T}$ to discriminate between CYP21A2 copies with c.955C $>\mathrm{T}$ in non-pathogenic and pathogenic genomic contexts can be readily built into molecular diagnosis approaches, ${ }^{32,33}$ which seems to be especially important for populations with high c. $955 \mathrm{C}>\mathrm{T}$ frequency from North Africa and Asia. ${ }^{34,35}$ Before its inclusion in the routine genetic diagnosis of $\mathrm{CAH}$, the occurrence pattern of $\mathrm{c}^{*} 12 \mathrm{C}>\mathrm{T}$ should be investigated in both healthy subjects and $\mathrm{CAH}$ patients belonging to the target population. The pioneer study on c.955C $>\mathrm{T}$ in RCCX structure with three segments and two CYP21A2 $2^{10}$ obviously illustrates the importance of validation in other populations: it is probable that the c. ${ }^{\star} 12 \mathrm{~T}$ and c. $293-13 \mathrm{G}$ CAH alleles are simultaneously harbored in the middle segment in three $\mathrm{CAH}$ patients. However, the nationality and ancestry of these patients have not been documented, and thus a conclusion with regard to the origin of this RCCX structure unfortunately cannot be drawn.

Nevertheless, the three CAH-causing mutations in one patient harboring $c^{*} 12 \mathrm{C}>\mathrm{T}$ implied that one of the mutations could occur in the CYP21A2 of the middle segment, disabling its functionality. Our evolutionary analysis provided a new insight into the underlying mechanism that generates such a clinical case. The CYP21A2 haplotypes with the c.955T allele are derived from the $3^{\prime}$-segment of the LBSASB RCCX structure or from CYP21A1P, according to the genealogical haplotype network. However, the second, functional copy of CYP21A2 in a chromosome was never carried by classical CAH patients with c.955C $>\mathrm{T}$, independent of whether the c.955T allele of CYP21A2 haplotypes was derived from the 3 '-segment of LBSASB or elsewhere. This putative rearrangement mechanism of CYP21A2 

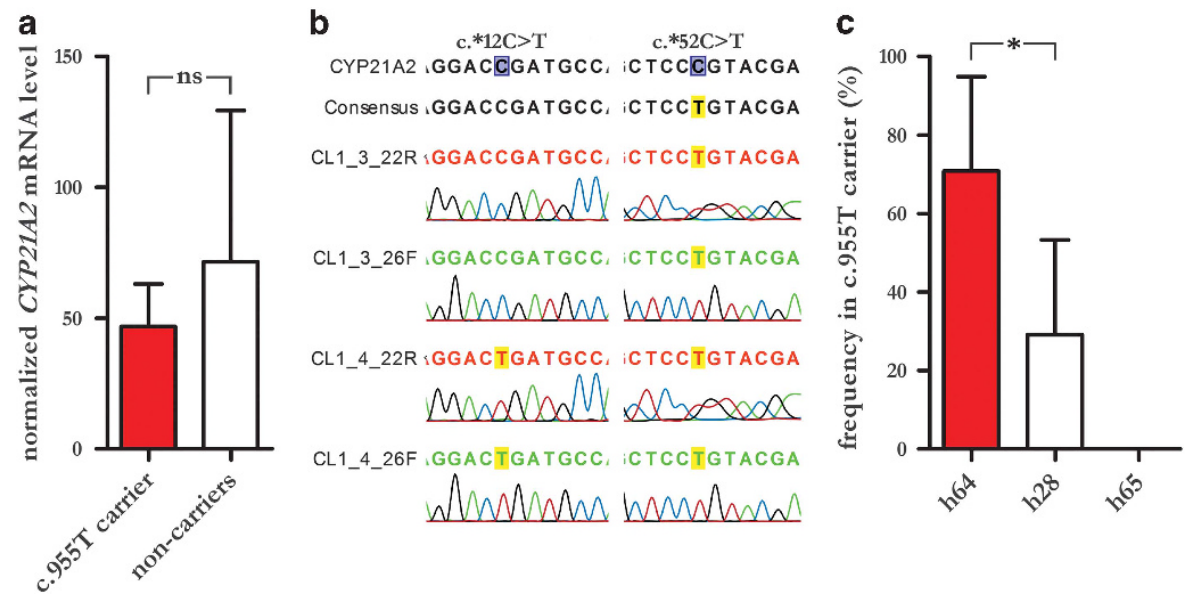

Figure 4 The results of the functional studies on adrenocortical specimens of NFAl patients. One asterisk indicates significant difference at $P<0.05$, whereas ns represents a nonsignificant difference, as calculated by $t$-test. (a) CYP21A2 mRNA level in the adrenal specimens of c.955T allele (on LBSASB RCCX structure) carrier NFAI patient $(N=1)$ and non-carrier patients $(N=3)$. (b) The forward and reverse chromatogram of h28 and h64 transcripts from the c.955T allele carrier NFAI patient, where the distinctive alleles on c. ${ }^{*} 12 \mathrm{C}>\mathrm{T}$ and $\mathrm{c} .{ }^{*} 52 \mathrm{C}>\mathrm{T}$ variants are indicated (CT alleles for $\mathrm{h} 28$ and TT alleles for h64). (c) The abundance of transcripts expressed from three CYP21A2 haplotypes (h28, h64, and h65) of the NFAI patient with c.955T allele on LBSASB RCCX structure.

haplotypes with the c.955T derived from the LBSASB RCCX structure sheds light on why the $\mathrm{c}^{*} 12 \mathrm{~T}$ allele did not occur in Hungarian $\mathrm{CAH}$ patients carrying a CYP21A2 haplotype with c.293-79A and c.955T; if a rearranged RCCX structure loses the functional CYP21A2 from the middle segment of LBSASB, it will become pathogenic, and if a rearranged RCCX structure retains the functionally intact CYP21A2 with $c^{*} 12 \mathrm{~T}$ from the middle segment, it will rescue its carrier from CAH. Nevertheless, it cannot be excluded that a rearrangement such as non-allelic gene conversion deletes or transfers the c. ${ }^{\star} 12 \mathrm{~T}$ allele from its original genomic context, or transfers a pathogenic mutation to CYP21A2 in the middle segment (the frequency of such an event will be very rare, because a rare event happens in the moderately frequent LBSASB). Therefore, careful consideration is always a basic requirement in the genetic diagnosis of $\mathrm{CAH}$, and the sequencing of full-length CYP21A2 from the segment-specific PCR product of the middle segment is needed after the finding of the $c^{*} 12 \mathrm{~T}$ allele in the extremely rare case when more than two $\mathrm{CAH}$ mutations (including c. $955 \mathrm{C}>\mathrm{T}$ ) are detected in one individual.

The significance of the $\mathrm{c} .955 \mathrm{C}>\mathrm{T}$ variant in the molecular diagnosis of $\mathrm{CAH}$ is testified by a recent case study on a misdiagnosed c. $955 \mathrm{C}>\mathrm{T}$ variant. ${ }^{36}$ This kind of case is not extremely rare, and there may be no better example of how true that is than one of our cases. The original genetic diagnosis of a 1-year-old patient with classical $\mathrm{CAH}$ was reexamined, because the required dose of hormone substitution was unchanged in spite of weight gain. The father had a known history of classical CAH due to CYP21A2 deficiency and the healthy mother carried a c.955T allele. The patient inherited the maternal c.955T allele besides a paternal RCCX structure without CYP21A2, and was therefore treated with mineralocorticoids and glucocorticoids from birth. The reexamination demonstrated that an intact CYP21A2 was harbored by the middle segment of LBSASB next to the $\mathrm{c} .955 \mathrm{C}>\mathrm{T}$ variant in the $3^{\prime}$-segment. The steroid supplementation had been gradually reduced, and then completely discontinued based on this finding, without any clinical signs of adrenocortical insufficiency.

Several studies based on the normal steroid levels of subjects with two carried $\mathrm{CAH}$ variants have found indirect evidence proving that one of the two CYP21A2 associated with the $\mathrm{c} .955 \mathrm{C}>\mathrm{T}$ variant in one chromosome expresses active steroid 21-hydroxylase enzyme. ${ }^{36-39}$ However, direct evidence for the functional CYP21A2 in the middle segment was provided by the current study for the first time. In the adrenal specimen of a patient with NFAI, the CYP21A2 in the middle segment of the LBSASB RCCX structure produced an mRNA transcript differing from the transcripts of the CYP21A2 copies in the 3 '-segment and the other chromosome, and this middle segmentspecific CYP21A2 transcript was detected. The CYP21A2 transcript from the middle segment was significantly more abundant than the other two transcripts, but neither CYP21A2 mRNA expression levels nor hormone levels in blood significantly differed between the LBSASB carriers and the non-carriers in NFAI patients. It should be noted that the quantitative analysis of the abundance of different CYP21A2 transcripts was not absolutely reliable because only one adrenal specimen was examined (however, we have no reason to question the existence of the CYP21A2 transcript expressed from the middle segment of LBSASB), and the sample size of NFAI patients was not sufficient for the detection of subtle hormonal differences in blood. In fact, several hundreds of adrenal specimens or NFAI patients should be examined to collect enough LBSASB carriers for the more reliable analyses on the abundance of CYP21A2 mRNAs or the subtle hormonal differences due to the moderate prevalence of LBSASB.

The current study is in tune with the results that have been presented so far (Table 2$)$. In addition, the allele frequencies $(\sim 1-2 \%)$ and the almost complete LD between c. $293-79 \mathrm{G}>\mathrm{A}$ and $\mathrm{c} .{ }^{*} 12 \mathrm{C}>\mathrm{T}$ in the European populations of the 1000 Genomes project ${ }^{40}$ also imply the presence of LBSASB RCCX structure. However, the nextgeneration sequencing (NGS)-based methods cannot reveal the exact internal content of multiallelic CNVs, ${ }^{41}$ and the bioinformatic analysis of NGS data is still prone to errors; ${ }^{42}$ hence, ${ }^{*}{ }^{\star} 12 \mathrm{C}>\mathrm{T}$ is documented by dbSNP as a variant of CYP21A2 in the $3^{\prime}$-segment. According to the current finding, the c. ${ }^{\star} 12$ nucleotide position is never polymorphic in the $3^{\prime}$-segment, but it is in the middle segment. Therefore, this phenomenon cannot be described by the traditional variant definition of non-duplicated genomic regions, and the usage of the variant categories for duplicated genes ${ }^{19,43}$ should be considered. 


\section{CONFLICT OF INTEREST}

The authors declare no conflict of interest.

\section{ACKNOWLEDGEMENTS}

We are grateful to Mark Eyre for English proofreading, and the technical assistance of Andrea Juhász is gratefully acknowledged. We thank the staff of Biomi Kft, Dr Adrienn Micsinai, Dr Anita Mohr, Dr Rita Sipos, and Réka Szántó-Egész for help with sequencing. This study was supported by Technology Innovation Fund, National Developmental Agency (KTIAAIK-2012-12-1-0010), and A Patócs is the recipient of a 'Lendulet' grant from the Hungarian Academy of Sciences.

1 Banlaki Z, Szabo JA, Szilagyi A et al: Intraspecific evolution of human RCCX copy number variation traced by haplotypes of the CYP21A2 gene. Genome Biol Evol 2013; 5: 98-112.

2 Handsaker RE, Van Doren V, Berman JR et al: Large multiallelic copy number variations in humans. Nat Genet 2015; 47: 296-303.

3 Blanchong CA, Zhou B, Rupert KL et al: Deficiencies of human complement component C4A and C4B and heterozygosity in length variants of RP-C4-CYP21-TNX (RCCX) modules in caucasians. The load of RCCX genetic diversity on major histocompatibility complex-associated disease. J Exp Med 2000; 191: 2183-2196.

4 Szilagyi A, Doleschall M, Fust G: Complement genes in the central region of the MHC. In: Mehra NK (ed): The HLA Complex in Biology and Medicine: A Resource Book. New Delhi, India: Jaypee Brothers Medical Publishers, 2010, pp 135-158.

5 Miller WL, Auchus RJ: The molecular biology, biochemistry, and physiology of human steroidogenesis and its disorders. Endocr Rev 2011; 32: 81-151.

6 White PC, Speiser PW: Congenital adrenal hyperplasia due to 21-hydroxylase deficiency. Endocr Rev 2000; 21: 245-291.

7 Cooper DN, Bacolla A, Ferec C, Vasquez KM, Kehrer-Sawatzki H, Chen JM: On the sequence-directed nature of human gene mutation: the role of genomic architecture and the local DNA sequence environment in mediating gene mutations underlying human inherited disease. Hum Mutat 2011; 32: 1075-1099.

8 New MI, Abraham M, Gonzalez B et al: Genotype-phenotype correlation in 1,507 families with congenital adrenal hyperplasia owing to 21-hydroxylase deficiency. Proc Natl Acad Sci USA 2013; 110: 2611-2616.

9 Wedell A: Molecular genetics of congenital adrenal hyperplasia (21-hydroxylase deficiency): implications for diagnosis, prognosis and treatment. Acta Paediatr 1998; 87: 159-164.

10 Wedell A, Stengler B, Luthman $\mathrm{H}$ : Characterization of mutations on the rare duplicated C4/CYP21 haplotype in steroid 21-hydroxylase deficiency. Hum Genet 1994; 94 : 50-54.

11 Witchel SS, Lee PA, Trucco M: Who is a carrier? Detection of unsuspected mutations in 21-hydroxylase deficiency. Am J Med Genet 1996; 61: 2-9.

12 Janner M, Pandey AV, Mullis PE, Fluck CE: Clinical and biochemical description of a novel CYP21A2 gene mutation 962_963insA using a new 3D model for the P450c21 protein. Eur J Endocrinol/Eur Fed Endocr Soc 2006; 155: 143-151.

13 Mohamed S, El-Kholy S, Al-Juryyan N, Al-Nemri AM, Abu-Amero KK: A CYP21A2 gene mutation in patients with congenital adrenal hyperplasia. Molecular genetics report from Saudi Arabia. Saudi Med J 2015; 36: 113-116.

14 Globerman H, Amor M, Parker KL, New MI, White PC: Nonsense mutation causing steroid 21-hydroxylase deficiency. J Clin Invest 1988; 82: 139-144.

15 Koppens PF, Hoogenboezem T, Degenhart HJ: CYP21 and CYP21P variability in steroid 21-hydroxylase deficiency patients and in the general population in the Netherlands. Eur J Hum Genet 2000; 8: 827-836.

16 Koppens PF, Hoogenboezem T, Degenhart HJ: Duplication of the CYP21A2 gene complicates mutation analysis of steroid 21-hydroxylase deficiency: characteristics of three unusual haplotypes. Hum Genet 2002; 111: 405-410.

17 Parajes S, Quinteiro C, Dominguez F, Loidi L: High frequency of copy number variations and sequence variants at CYP21A2 locus: implication for the genetic diagnosis of 21hydroxylase deficiency. PLOS ONE 2008; 3: e2138.

18 Kleinle S, Lang R, Fischer GF et al: Duplications of the functional CYP21A2 gene are primarily restricted to Q318X alleles: evidence for a founder effect. J Clin Endocrinol Metab 2009; 94: 3954-3958.
19 Szabo JA, Szilagyi A, Doleschall Z et al: Both positive and negative selection pressures contribute to the polymorphism pattern of the duplicated human CYP21A2 gene. PLoS ONE 2013; 8: e81977.

20 Young WFJr: Clinical practice. The incidentally discovered adrenal mass. N Engl J Med 2007; 356: 601-610.

21 Banlaki Z, Doleschall M, Rajczy K, Fust G, Szilagyi A: Fine-tuned characterization of RCCX copy number variants and their relationship with extended MHC haplotypes. Genes Immun 2012; 13: 530-535.

22 Doleschall M, Szabo JA, Pazmandi J et al: Common genetic variants of the human steroid 21-hydroxylase gene (CYP21A2) are related to differences in circulating hormone levels. PLOS ONE 2014; 9: e107244.

23 Luczay A, Torok D, Ferenczi A, Majnik J, Solyom J, Fekete G: Potential advantage of N363S glucocorticoid receptor polymorphism in 21-hydroxylase deficiency. Eur $J$ Endocrinol/Eur Fed Endocr Soc 2006; 154: 859-864.

24 Patocs $A$, Toth M, Barta $C$ et al: Hormonal evaluation and mutation screening for steroid 21-hydroxylase deficiency in patients with unilateral and bilateral adrenal incidentalomas. Eur J Endocrinol/Eur Fed Endocr Soc 2002; 147: 349-355.

25 Boyle B, Butz H, Liko I et al: Expression of glucocorticoid receptor isoforms in human adrenocortical adenomas. Steroids 2010; 75: 695-700.

26 Cervenak J, Doleschall M, Bender B et al: NFkappaB induces overexpression of bovine FcRn: a novel mechanism that further contributes to the enhanced immune response in genetically modified animals carrying extra copies of FcRn. mAbs 2013; 5: 860-871.

27 de Jonge HJ, Fehrmann RS, de Bont ES et al: Evidence based selection of housekeeping genes. PLOS ONE 2007; 2: e898.

28 Doleschall M, Mayer B, Cervenak J, Cervenak L, Kacskovics I: Cloning, expression and characterization of the bovine p65 subunit of NFkappaB. Dev Comp Immunol 2007; 31: 945-961.

29 Posada D, Crandall KA: Intraspecific gene genealogies: trees grafting into networks. Trends Ecol Evol 2001; 16: 37-45.

30 Chung EK, Yang Y, Rennebohm RM et al: Genetic sophistication of human complement components C4A and C4B and RP-C4-CYP21-TNX (RCCX) modules in the major histocompatibility complex. Am J Hum Genet 2002; 71: 823-837.

31 Concolino P, Mello E, Minucci A, Giardina B, Capoluongo E: Genes, pseudogenes and like genes: the case of 21-hydroxylase in Italian population. Clin Chim Acta 2013; 424: 85-89.

32 Tsai LP, Cheng CF, Chuang SH, Lee HH: Analysis of the CYP21A1P pseudogene: indication of mutational diversity and CYP21A2-like and duplicated CYP21A2 genes. Anal Biochem 2011; 413: 133-141.

$33 \mathrm{Xu} \mathrm{Z}$, Chen W, Merke DP, McDonnell NB: Comprehensive mutation analysis of the CYP21A2 gene: an efficient multistep approach to the molecular diagnosis of congenital adrenal hyperplasia. J Mol Diagn 2013; 15: 745-753.

34 Kharrat M, Tardy V, M'Rad R et al: Molecular genetic analysis of Tunisian patients with a classic form of 21-hydroxylase deficiency: identification of four novel mutations and high prevalence of Q318X mutation. J Clin Endocrinol Metab 2004; 89: 368-374.

35 Marumudi E, Sharma A, Kulshreshtha B, Khadgawat R, Khurana ML, Ammini AC: Molecular genetic analysis of CYP21A2 gene in patients with congenital adrenal hyperplasia. Indian J Endocrinol Metab 2012; 16: 384-388.

36 Lekarev $\mathrm{O}$, Tafuri K, Lane $\mathrm{AH}$ et al: Erroneous prenatal diagnosis of congenital adrenal hyperplasia owing to a duplication of the CYP21A2 gene. J Perinato/ 2013; 33: 76-78.

37 Baumgartner-Parzer SM, Nowotny P, WaldhausI W, Vierhapper H: A rare duplicated 21hydroxylase haplotype and a de novo mutation: a family analysis. J Clin Endocrinol Metab 2003; 88: 2794-2796.

38 Kharrat M, Riahi A, Maazoul F, M'Rad R, Chaabouni H: Detection of a frequent duplicated CYP21A2 gene carrying a Q318X mutation in a general population with quantitative PCR methods. Diagn Mol Pathol Part B 2011; 20: 123-127.

39 Leccese A, Longo V, Dimatteo $C$ et al: Lack of genotypephenotype correlation in congenital adrenal hyperplasia due to a CYP21A2-like gene. Clin Chim Acta 2014; 437: 48-51.

40 Auton A, Brooks LD, Durbin RM et al: A global reference for human genetic variation. Nature 2015; 526: 68-74.

41 Nuttle X, Huddleston J, O'Roak BJ et al: Rapid and accurate large-scale genotyping of duplicated genes and discovery of interlocus gene conversions. Nat Methods 2013; 10: 903-909.

$42 \mathrm{Li} \mathrm{H}$ : Toward better understanding of artifacts in variant calling from high-coverage samples. Bioinformatics 2014; 30: 2843-2851.

43 Innan H: Population genetic models of duplicated genes. Genetica 2009; 137: 19-37.

Supplementary Information accompanies this paper on European Journal of Human Genetics website (http://www.nature.com/ejhg) 